\title{
Effects of Rapid Response Systems on Clinical Outcomes: Systematic Review and Meta-Analysis
}

\author{
Sumant R. Ranji, MD ${ }^{1}$ \\ Andrew D. Auerbach, MD, MPH ${ }^{1}$ \\ Caroline J. Hurd, $\mathrm{MD}^{2}$ \\ Keith O'Rourke, PhD ${ }^{3}$ \\ Kaveh G. Shojania, $\mathrm{MD}^{3}$ \\ ${ }^{1}$ Department of Medicine, University of California \\ San Francisco, San Francisco, California \\ ${ }^{2}$ Department of Medicine, University of Washing- \\ ton, Seattle, Washington \\ ${ }^{3}$ Department of Medicine, University of Ottawa \\ and Ottawa Health Research Institute, Ottawa, On- \\ tario, Canada
}

BACKGROUND: A rapid response system (RRS) consists of providers who immediately assess and treat unstable hospitalized patients. Examples include medical emergency teams and rapid response teams. Early reports of major improvements in patient outcomes led to widespread utilization of RRSs, despite the negative results of a subsequent cluster-randomized trial.

PURPOSE: To evaluate the effects of RRSs on clinical outcomes through a systematic literature review.

DATA SOURCES: MEDLINE, BIOSIS, and CINAHL searches through August 2006, review of conference proceedings and article bibliographies.

STUDY SELECTION: Randomized and nonrandomized controlled trials, interrupted time series, and before-after studies reporting effects of an RRS on inpatient mortality, cardiopulmonary arrests, or unscheduled ICU admissions.

DATA EXTRACTION: Two authors independently determined study eligibility, abstracted data, and classified study quality.

DATA SYNTHESIS: Thirteen studies met inclusion criteria: 1 cluster-randomized controlled trial (RCT), 1 interrupted time series, and 11 before-after studies. The RCT showed no effects on any clinical outcome. Before-after studies showed reductions in inpatient mortality $(\mathrm{RR}=0.82,95 \% \mathrm{CI}$ : 0.74-0.91) and cardiac arrest $(\mathrm{RR}=0.73,95 \% \mathrm{CI}: 0.65-0.83)$. However, these studies were of poor methodological quality, and control hospitals in the RCT reported reductions in mortality and cardiac arrest rates comparable to those in the before-after studies.

CONCLUSIONS: Published studies of RRSs have not found consistent improvement in clinical outcomes and have been of poor methodological quality. The positive results of before-after trials likely reflects secular trends and biased outcome ascertainment, as the improved outcomes they reported were of similar magnitude to those of the control group in the RCT. The effectiveness of the RRS concept remains unproven. Journal of Hospital Medicine 2007;2:422-432. (C) 2007 Society of Hospital Medicine.

KEYWORDS: systematic review, rapid response systems.

A medical emergency team ${ }^{1}$ is a group of clinicians trained to A quickly assess and treat hospitalized patients showing acute signs of clinical deterioration. Equivalent terms used are rapid response team, ${ }^{2}$ critical care outreach team, ${ }^{3}$ and patient-at-risk team. ${ }^{4}$ A consensus panel ${ }^{5}$ recently endorsed use of the term rapid response system (RRS) to denote any system that uses a standard set of clinical criteria to summon caregivers to the bedside of a patient who is deemed unstable but not in cardiopulmonary arrest (in which case a standard resuscitation team would be summoned). Such teams primarily evaluate patients on general hospital wards.

RRSs have been developed in response to data indicating that patients frequently demonstrate premonitory signs or receive inadequate care prior to unanticipated intensive care unit (ICU)
The authors thank Emmanuel King, MD, for graciously providing a copy of his manuscript prior to publication and Alexis Meredith, MD, for providing additional information regarding his study. Dr. Shojania holds a Government of Canada Research Chair in Patient Safety and Quality Improvement. 
Example of Rapid Response System Calling Criteria for Adult Patients

Any staff member may call the team if 1 of the following criteria is met:

- Heart rate $>140 / \mathrm{min}$ or $<40 / \mathrm{min}$

- Respiratory rate $>28 / \mathrm{min}$ or $<8 / \mathrm{min}$

- Systolic blood pressure $>180 \mathrm{mmHg}$ or $<90 \mathrm{~mm} \mathrm{Hg}$

- Oxygen saturation $<90 \%$ despite supplementation

- Acute change in mental status

- Urine output $<50$ cc over 4 hours

- Staff member has significant concern about patient's condition

Additional criteria used at some institutions:

- Chest pain unrelieved by nitroglycerin

- Threatened airway

- Seizure

- Uncontrolled pain

admission, cardiopulmonary arrest, or death outside the ICU. ${ }^{6-14}$ Earlier identification and treatment of such patients could prevent adverse clinical outcomes. The structure of RRSs varies but generally includes a physician and nurse and may also include other staff such as respiratory therapists. ${ }^{5}$ Teams are summoned by hospital staff to assess patients meeting specific clinical criteria (see box) about whom the bedside staff has significant concern. ${ }^{15}$

Initial studies of RRSs, performed primarily in Australia and the United Kingdom, showed promising reductions in unanticipated ICU admissions, cardiac arrests, and even overall inpatient mortality. ${ }^{1,16,17}$ The considerable enthusiasm generated by these studies ${ }^{18,19}$ resulted in the Institute for Healthcare Improvement (IHI) incorporating RRSs into its "100,000 Lives" campaign, ${ }^{2}$ and RRSs are now being implemented in the more than 3000 U.S. hospitals that joined the campaign. However, a recent commentary on rapid response teams ${ }^{20}$ and a systematic review of critical care outreach teams ${ }^{21}$ have raised concerns that this widespread implementation may not be justified by the available evidence. We performed a systematic review of studies of all variations of RRSs in order to determine their effect on patient outcomes and to characterize variations in their organization and implementation.

\section{METHODS}

\section{Literature Search and Inclusion and Exclusion Criteria}

We systematically searched MEDLINE, CINAHL, and BIOSIS through August 2006 for relevant studies using the various terms for RRSs (eg, "medical emergency team," "rapid response team," "critical care outreach") and medical subject headings rele- vant to inpatient care and critical illness (eg, "patient care team" and "resuscitation"; the full search strategy is given in the Appendix). We also reviewed the abstract lists from the 2004 and 2005 American Thoracic Society and Society of Critical Care Medicine annual meetings and scanned reference lists from key articles.

We screened the abstracts of the articles identified by the search, and 2 independent reviewers abstracted potentially relevant articles using a standardized data abstraction form. Disagreements between the reviewers were resolved by consensus and, if necessary, discussion with a third reviewer. We included randomized controlled trials (RCTs), controlled before-after studies, and interrupted time series, including simple before-after studies with no contemporaneous control group, though we planned to separately analyze data from controlled studies if possible. We included only English-language articles.

On the basis of RRS features in widely cited articles $^{22-24}$ and the recommendations of a recent consensus statement, ${ }^{5}$ we defined an RRS as having the following characteristics: (1) its primary responsibility is to intervene whenever hospitalized patients become unstable before cardiopulmonary arrest occurs; (2) it must primarily provide care outside the ICU and emergency department; (3) specific clinical criteria must be in place that define instability and trigger a call to the team; and (4) it must be expected to respond within a specified time. We defined these criteria in order to distinguish studies of RRSs from studies of cardiac arrest ("code blue") teams or traditional consulting services.

To be included in the analysis, articles had to report the effects of a rapid response system on at least 1 of these outcomes: inpatient mortality, inpatient cardiac arrest, or unscheduled ICU transfer. We used the definitions of cardiac arrest and unscheduled ICU transfer given in the primary studies. In addition to these outcomes, we abstracted information on the number of admissions and the number of RRS calls during the study period. To maximize the comparability of study outcomes, we calculated the rates of mortality, cardiac arrest, unscheduled ICU transfer, and RRS calls per 1000 admissions for studies that did not supply data in this fashion.

\section{Assessment of Study Quality}

Quality scoring instruments for studies included in systematic reviews generally focus on randomized 
TABLE 1

Study Quality Criteria

Quality measures
A. Internal validity
1. Did the study have a contemporaneous control group?
2. If there was no contemporaneous control group, did the study report data
for more than 1 time point before and after the intervention?
3. Were nonobjective primary outcomes (eg, unplanned ICU transfer)
measured in a blinded fashion?
4. Were patients made DNR by the RRS included in calculations of the cardiac
arrest and mortality rates?
B. Generalizability
5. Was the intervention performed independent of other quality improvement
interventions targeting the care of critically ill patients?
6. Did the study report the number of admissions and RRS calls during the
study period?
7. Did the study report the availability of intensivists before and after the
intervention?

Elements affecting study internal validity and translatability. These elements were chosen based on the methods of the Cochrane collaboration. ${ }^{25}$ These criteria were not used to determine article inclusion or exclusion.

controlled trials, which we anticipated would account for a minority of included studies. On the basis of recommendations for the assessment of methodology for nonrandomized study designs, ${ }^{25,26}$ we identified and abstracted 4 important determinants of internal validity (Table 1). The consensus statement ${ }^{5}$ recommends monitoring the effectiveness of RRSs by measuring the rate of unscheduled ICU admissions (defined as an unplanned admission to the ICU from a general ward $^{27}$ ) and cardiac arrests of patients who were not listed as "do not resuscitate" (DNR). As the definition of unscheduled ICU admission allows room for subjectivity, we considered the blinding of assessment of this outcome to study group assignment to be important, especially for retrospective studies. Measurement of cardiac arrests should be less susceptible to blinding issues, but one of the functions of an RRS can be to initiate discussions that result in changes in the goals of care and code status. $^{22}$ Thus, excluding patients made DNR by the team from cardiac arrest calculations could falsely lower the cardiac arrest rate.

We also abstracted 3 separate elements of study quality pertaining to the external validity or generalizability of included studies (Table 1). These elements were defined a priori by consensus reached by discussion among the reviewers. These elements were intended to provide a framework for interpreting the included studies and to guide subgroup analyses. They were not used to form a composite quality score.

\section{Statistical Analysis}

We performed a random-effects meta-analysis to calculate summary risk ratios with $95 \%$ confidence intervals for the effects of RRSs on inpatient mortality, cardiopulmonary arrest, and unscheduled ICU admission. Included in the meta-analysis were the studies that reported total number of admissions and incidence of each outcome before and after institution of the RRS. For randomized trials that reported pre- and postintervention data, we treated the intervention and control groups as separate trials in order to be able to compare their effects with the before-after trials. For studies that reported results adjusted for clustering (ie, by hospital), we back-calculated the unadjusted results by multiplying the standard error by the square of the design factor coefficient. ${ }^{28,29}$ We calculated the $I^{2}$ statistic to assess heterogeneity. ${ }^{30}$ All analyses were performed using Stata version 8.2 (Stata Corporation, College Station, TX).

\section{RESULTS}

The database searches identified 861 citations, and 1 additional prepublication study was supplied by the study's first author ${ }^{31} ; 89$ articles underwent fulltext review (Fig. 1). Most studies excluded during the full-text review did not meet our criteria for a study of an RRS or were observational studies or review articles. For instance, Sebat et al. ${ }^{32}$ published a study of a "shock team" at a community hospital that intervened when any patient suffered nontraumatic shock; the study did not meet our inclusion criteria as all patients were admitted to the ICU, most directly from the emergency department. Another frequently cited study, by Bristow et al., ${ }^{16}$ was excluded as it was a case-control study.

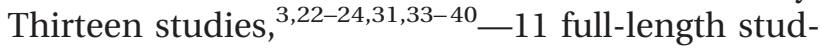
ies and 2 abstracts-met all criteria for inclusion.

\section{Characteristics of Included Trials}

The characteristics of included studies are outlined in Table 2. Five studies were performed in Australia, 4 in the United States, and 4 in the United Kingdom. All were conducted in academic teaching hospitals. Two studies ${ }^{37,38}$ focused on pediatric inpatients, and the remainder involved hospitalized adults. The RRS intervened for all hospitalized patients in all but 2 studies ( 1 of which focused on surgical inpatients ${ }^{33}$ and the other in which the RRS 


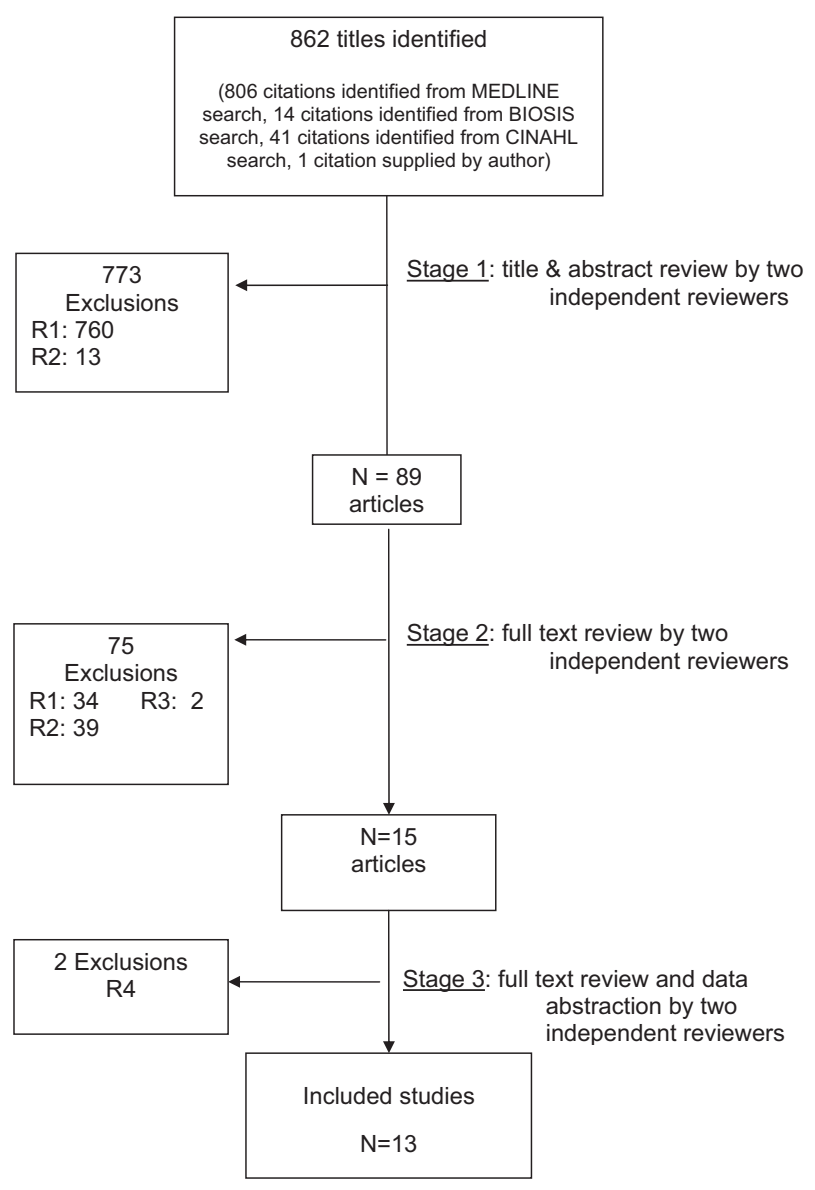

FIGURE 1. Article identification and triage trial flow diagram, as recommended by the QUOROM statement for improving the methodological quality of systematic reviews and meta-analyses. ${ }^{54}$ Reasons for exclusion were: R1, not a study of a rapid response system; R2, ineligible study design (not simple before-after study, controlled before-after study, interrupted time series, or randomized, controlled trial); R3, no eligible outcomes (did not report effect of RRS on in-hospital cardiac arrest, unscheduled ICU admission, or inpatient mortality); R4, overlapping publication. Data from 1 article ${ }^{55}$ were pooled with an included article, ${ }^{34}$ and the other ${ }^{56}$ was excluded because it contained longer-term follow-up data from another included study. ${ }^{23}$

evaluated only patients discharged from the $\mathrm{ICU}^{3}$ ). In 2 studies, ${ }^{31,39}$ the RRS was available to evaluate outpatients, such as hospital visitors, in addition to inpatients.

\section{RRS Structure, Calling Criteria, and Responsibilities}

Seven studies ${ }^{22,23,31,33,34,36,37}$ that described the team composition used variants of the medical emergency team model, a physician-led team (Table 2). In 6 of these 7 studies, the team included a critical care physician (attending or fellow) and an
ICU nurse; in the sole RCT (the MERIT study ${ }^{36}$ ), the team structure varied between hospitals, consisting of a nurse and physician from either the emergency department or ICU. Hospitalists, who are involved in RRS responses at many U.S. hospitals, were primary team leaders of the RRS in only 1 study. ${ }^{31}$ In 2 studies $^{34,37}$ the RRS also responded to code blue calls, and in 4 studies ${ }^{23,31,33,39}$ the RRS and the code blue team had separate personnel; the remaining studies did not define the distinction between RRS and code blue team.

In 4 studies the RRSs were led by nurses. One study published in abstract form ${ }^{39}$ used the rapid response team model, consisting of a critical care nurse and a respiratory therapist, with assistance as needed from the primary medical staff and a critical care physician. Three studies ${ }^{3,24,35}$ from UK hospitals used the critical care outreach (CCO) model, in which ICU-trained nurses respond initially with assistance from intensivists. The CCO model also involves follow-up on patients discharged from the ICU and proactive rounding on unstable ward patients.

The hospitals used broadly similar approaches to determining when to summon the RRS , relying on combinations of objective clinical criteria (eg, vital sign abnormalities) and subjective criteria (eg, acute mental status change, staff member concerned about patient's condition). Three studies $^{3,24,35}$ used a formal clinical score (the PatientAt-Risk score or the Modified Early Warning score) to trigger calls to the RRS. Three studies, 2 of them from the same institution, ${ }^{23,33}$ reported the frequency of specific triggers for RRS activation. Concern by bedside staff and respiratory distress were the most frequent activators of the RRS.

\section{Study Internal Validity and Generalizability}

One study, ${ }^{36}$ the MERIT trial, conducted in Australia, was a cluster-randomized RCT (randomized by hospital) that adhered to recommended elements of design and reporting for studies of this type. ${ }^{41} \mathrm{In}$ this study, hospitals in the control group received an educational intervention on caring for deteriorating patients only; hospitals in the intervention group received the educational module and started an RRS. An additional study ${ }^{35}$ identified itself as a randomized trial, but randomization occurred at the hospital ward level, with introduction of the intervention (critical care outreach) staggered so that at different points an individual ward could have been in either the control or intervention group; therefore, 


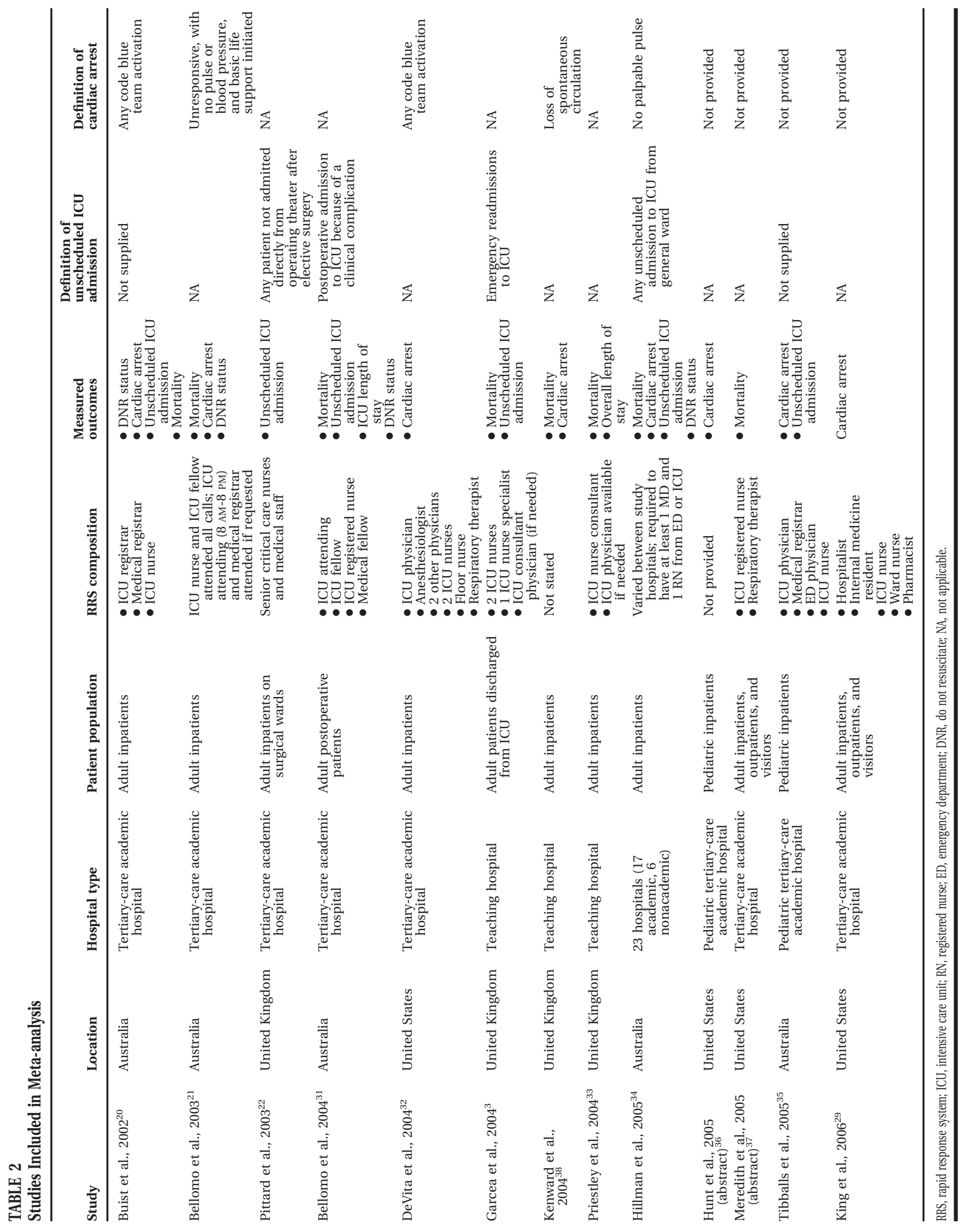


TABLE 3

Factors Affecting Internal Validity and Generalizability of Studies Included in Meta-analysis

\begin{tabular}{|c|c|c|c|c|c|c|c|}
\hline Study & $\begin{array}{l}\text { Contemporaneous } \\
\text { control group }\end{array}$ & $\begin{array}{l}\text { Data reported at more } \\
\text { than } 1 \text { time before/ } \\
\text { after intervention }\end{array}$ & $\begin{array}{l}\text { RRS calling } \\
\text { rate reported }\end{array}$ & $\begin{array}{l}\text { Outcomes analysis } \\
\text { included patients } \\
\text { made DNR by team }\end{array}$ & $\begin{array}{l}\text { Blind measurement of } \\
\text { nonobjective outcomes }\end{array}$ & $\begin{array}{l}\text { Intensivist always } \\
\text { available }\end{array}$ & $\begin{array}{l}\text { Other QI efforts } \\
\text { during study }\end{array}$ \\
\hline Buist et al., 2002²0 & No & No & Yes & № (mortality) & No & $\mathrm{NR}$ & NR \\
\hline Bellomo et al., $2003^{21}$ & No & No & Yes & Yes (mortality) & NA & Yes (ICU fellow) & No \\
\hline Pittard et al., $2003^{22}$ & No & No & Yes & $\mathrm{NA}$ & No & NR & NR \\
\hline Bellomo et al., $2004^{31}$ & No & No & Yes & Yes (mortality) & No & Yes (ICU fellow) & No \\
\hline DeVita et al., $2004^{32}$ & No & Yes & Yes & $\mathrm{NA}$ & No & $\begin{array}{l}\text { Yes (critical care } \\
\text { attending } \\
\text { physician) }\end{array}$ & NR \\
\hline Garcea et al., $2004^{33}$ & No & No & No & Unclear & No & $\mathrm{NR}$ & NR \\
\hline Kenward et al., $2004^{38}$ & No & No & Yes & Unclear & No & NR & NR \\
\hline Priestley et al., $2004^{33}$ & $\begin{array}{l}\text { No (interrupted time } \\
\text { series) }\end{array}$ & Yes & No & $\mathrm{NA}$ & No & NR & NR \\
\hline Hillman et al., $2005^{34}$ & Yes & No & Yes & Unclear & Yes & NR & No \\
\hline $\begin{array}{l}\text { Hunt et al., } 2005 \\
\text { (abstract) }\end{array}$ & No & No & Yes & $\mathrm{NA}$ & $\mathrm{NR}$ & NR & NR \\
\hline $\begin{array}{l}\text { Meredith et al., } 2005 \\
\quad \text { (abstract) }^{37}\end{array}$ & No & No & Yes & No & $\mathrm{NA}$ & No & No \\
\hline Tibballs et al., $2005^{35}$ & No & No & Yes & Unclear & No & NR & $\begin{array}{c}\text { Yes (educational workshops/ } \\
\text { more training in APLS) }\end{array}$ \\
\hline King et al., $2006^{29}$ & No & Yes & Yes & $\mathrm{NA}$ & No & Yes & No \\
\hline
\end{tabular}

SBA, simple before-after (quasi-experimental) study; ITS, interrupted time series; RCT, randomized controlled trial; NA, not applicable; NR, not reported; APLS, advanced pediatric life support

this study was considered an interrupted time series. All other trials included were before-after studies with no contemporaneous control group

Most studies did not meet criteria for internal validity or generalizability (Table 2 ). Two studies s.35 $^{3,3}$ did not report the number of RRS calls during the study period. One study ${ }^{22}$ omitted patients whose resuscitation status was changed after RRS evaluation from the calculation of inpatient mortality; thus, the patients who had been made "do not resuscitate" by the RRS did not contribute to the calculated mortality rate. The disposition of these patients was unclear in another study. ${ }^{36}$ All studies measured clinical outcomes retrospectively, and no studies reported blinding of outcomes assessors for nonobjective outcomes (eg, unplanned ICU admission). Studies generally did not report on the availability of intensivists or if other quality improvement interventions targeting critically ill patients were implemented along with the RRS.

\section{RRS Usage and Effects on Patient Outcomes}

Seven studies ${ }^{22-24,34,36-38}$ reported enough information to calculate the RRS calling rate (4 studies $^{24,31,39,40}$ reported the total number of calls but not the number of admissions, and 2 studies $^{3,35}$ did not report either). In these 7 studies, the calling rate varied from 4.5 to 25.8 calls per 1000 admissions. Three studies documented the calling rate before and after the intervention: a study at a hospital with a preexisting RRS $^{34}$ reported that the calling rate increased from 13.7 to 25.8 calls per 1000 admissions after an intensive education and publicity program; in a pediatric trial, ${ }^{38}$ the overall emergency calling rate (for cardiac arrests and medical emergencies) was reported to increase from 6.6 to 10.4 per 1000 admissions; and in the MERIT trial, ${ }^{36}$ calls increased from 3.1 to 8.7 per 1000 admissions.

\section{Effects of RRS on Clinical Outcomes}

Nine studies $3,22,23,33,35-37,39,40$ reported the effect of an RRS on inpatient mortality, 9 studies $^{22,23,31,33,34,36-38,40}$ reported its effect on cardiopulmonary arrests, and 6 studies $^{3,22,24,33,36,37}$ reported its effect on unscheduled ICU admissions. Of these, 7 trials that reported mortality and cardiopulmonary arrests and 6 studies that reported unscheduled ICU admissions supplied sufficient data for meta-analysis.

Observational studies demonstrated improvement in inpatient mortality, with a summary risk ratio of 0.82 (95\% CI: $0.74-0.91$, heterogeneity $I^{2}$ 


\begin{tabular}{|c|c|c|c|c|c|c|}
\hline Randomized trials & & & $\begin{array}{l}\text { Relative Risk } \\
(95 \% \mathrm{Cl})\end{array}$ & $\begin{array}{c}\text { Patient } \\
\text { population }\end{array}$ & $\begin{array}{c}\text { Calls } / 1000 \\
\text { admits }\end{array}$ & $\begin{array}{c}\text { Mortality/1000 } \\
\text { admits at } \\
\text { baseline }\end{array}$ \\
\hline MERT, 2005 (intervention) & - & & $0.65(0.48,0.88)$ & Adult & 8.7 & 1.65 \\
\hline MERT, 2005 (control) & $\square$ & & $0.73(0.53,1.02)$ & Adult & 3.1 & 1.60 \\
\hline Subtotal & $\infty$ & & $0.69(0.55,0.86)$ & & & \\
\hline \multicolumn{7}{|l|}{ Observational trials } \\
\hline Garcea, 2004 & - & & $0.52(0.35,0.77)$ & Adult & NR & NR \\
\hline Bellomo, 2004 & $=$ & & $0.59(0.41,0.86)$ & Adult & 4.2 & 6.03 \\
\hline Bellomo, 2003 & & & $0.83(0.68,1.00)$ & Adult & 5.6 & 25.5 \\
\hline Buist, 2002 & & & $0.90(0.79,1.04)$ & Adult & 5.3 & 15.1 \\
\hline Tibballs, 2005 & & & $0.45(0.10,1.99)$ & Pediatric & 5.1 & 0.12 \\
\hline \multirow[t]{3}{*}{ Subtotal } & $\diamond$ & & $0.82(0.74,0.91)$ & & & \\
\hline & 1 & $T$ & 1 & & & \\
\hline & $\begin{array}{l}.5 \\
\text { Relat } \\
\text { uced }\end{array}$ & 5 & 10 & & & \\
\hline
\end{tabular}

FIGURE 2. Effect of RRS on inpatient mortality The forest plot compares the relative risk of mortality after implementation of RRS with that before RRS implementation. For the MERIT trial, we treated the 2 study arms (intervention and control) as separate before-after trials in order to compare with the observational studies. The study by Garcea et al. ${ }^{3}$ evaluated the effect of RRS on readmission to the ICU. The supplied outcomes are for in-hospital mortality of patients readmitted to the ICU only; thus, the baseline mortality rate is not reported. The study by Bellomo et al. (2004) ${ }^{33}$ evaluated the effect of RRS on postoperative patients only. The other study performed at the same institution and published in $2003^{23}$ reported outcomes of all inpatients. Therefore, we subtracted the results of the 2004 study from those reported in the 2003 study to avoid counting the same outcomes twice (RR, relative risk; NR, not reported; NA, not applicable).

62.1\%; Fig. 2). However, the magnitude of these improvements was very similar to that seen in the control group of the MERIT trial (RR 0.73, 95\% CI: 0.53-1.02). The intervention group of the MERIT trial also demonstrated a reduction in mortality that was not significantly different from that of the control group (RR 0.65, 95\% CI: 0.48-0.87). We found a similar pattern in studies reporting RRS effects on cardiopulmonary arrests (Fig. 3). The observational studies did not show any effect on the risk of unscheduled ICU admissions (summary RR 1.08, 95\% CI: 0.96-1.22, heterogeneity $I^{2} 79.1 \%$ ) nor did the MERIT trial (Fig. 4).

\section{DISCUSSION}

Despite the strong face validity of the RRS concept, the current literature on medical emergency teams, rapid response teams, and critical care outreach suffers from substantial flaws that make it difficult to determine the effect of an RRS on patient outcomes. These flaws include the use of suboptimal study designs, failure to report important cointerventions, the methods in which outcomes were defined, and lack of verification of the validity of the outcomes measured. As a result, very little empiric data are available to define the effectiveness of
RRSs or to provide guidance for hospitals planning to implement an RRS.

Though early studies reported that RRSs appeared to reduce mortality and cardiac arrest rates, the sole randomized trial of an RRS (the MERIT trial $^{36}$ ) showed no differences between intervention and control hospitals for any clinical outcome. Both inpatient mortality and cardiac arrest rates declined in the intervention and control groups of the MERIT trial, and the reductions in these outcomes in observational trials were similar to those seen in the MERIT control group. This strongly implies that other factors besides the RRS were responsible for the results of previous before-after studies. These studies, which have been widely cited by proponents of the RRS, suffer from methodological limitations intrinsic to the study design and issues with outcome measurement that may have introduced systematic bias; these factors likely explain the contrast between the generally positive results of the before-after studies and the negative results of the MERIT trial.

Most early RRS trials used an uncontrolled before-after study design, as is common in quality improvement studies. ${ }^{42}$ This study design cannot account for secular trends or other factors, includ- 


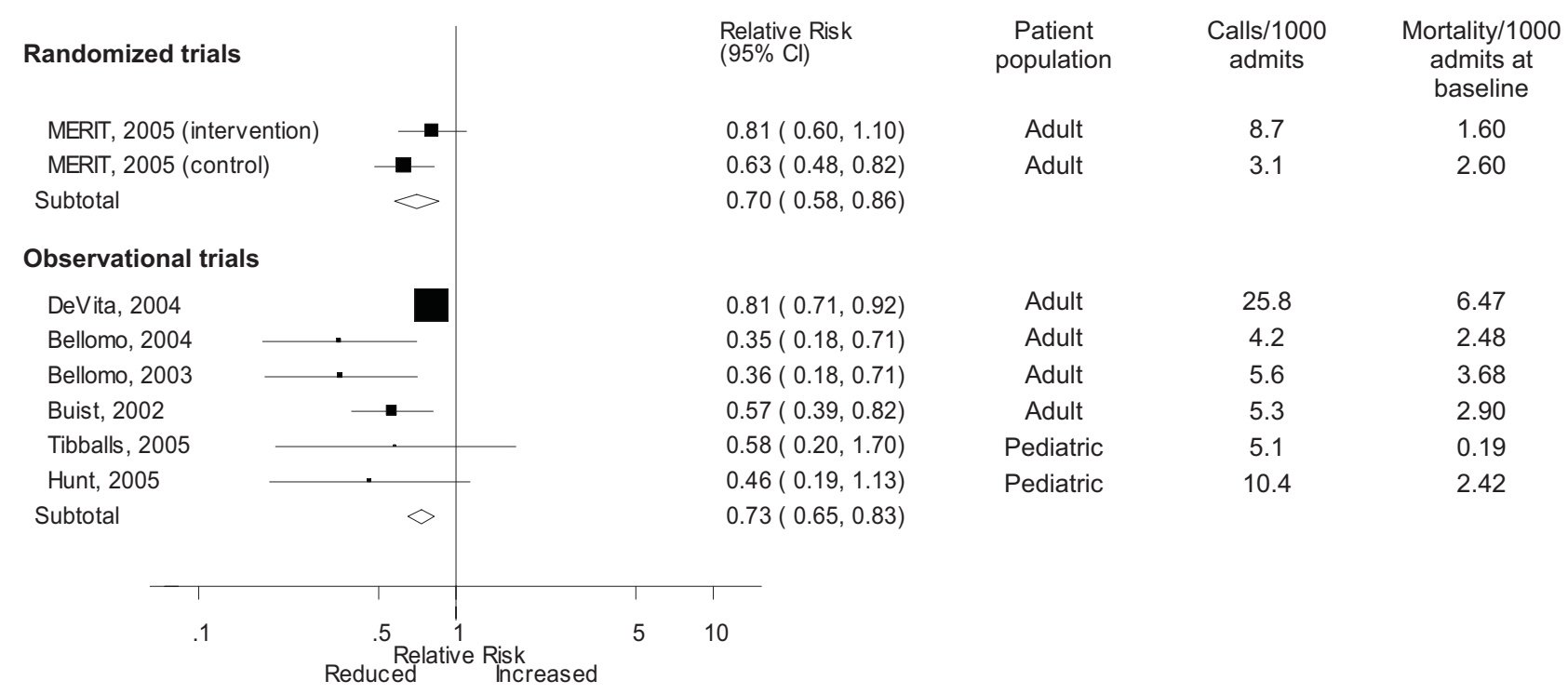

FIGURE 3. Effect of RRS on cardiopulmonary arrests The forest plot shows the relative risk of cardiopulmonary arrest after implementation of RRS. As in Figure 1, the MERIT trial intervention and control groups were treated as separate before-after trials.

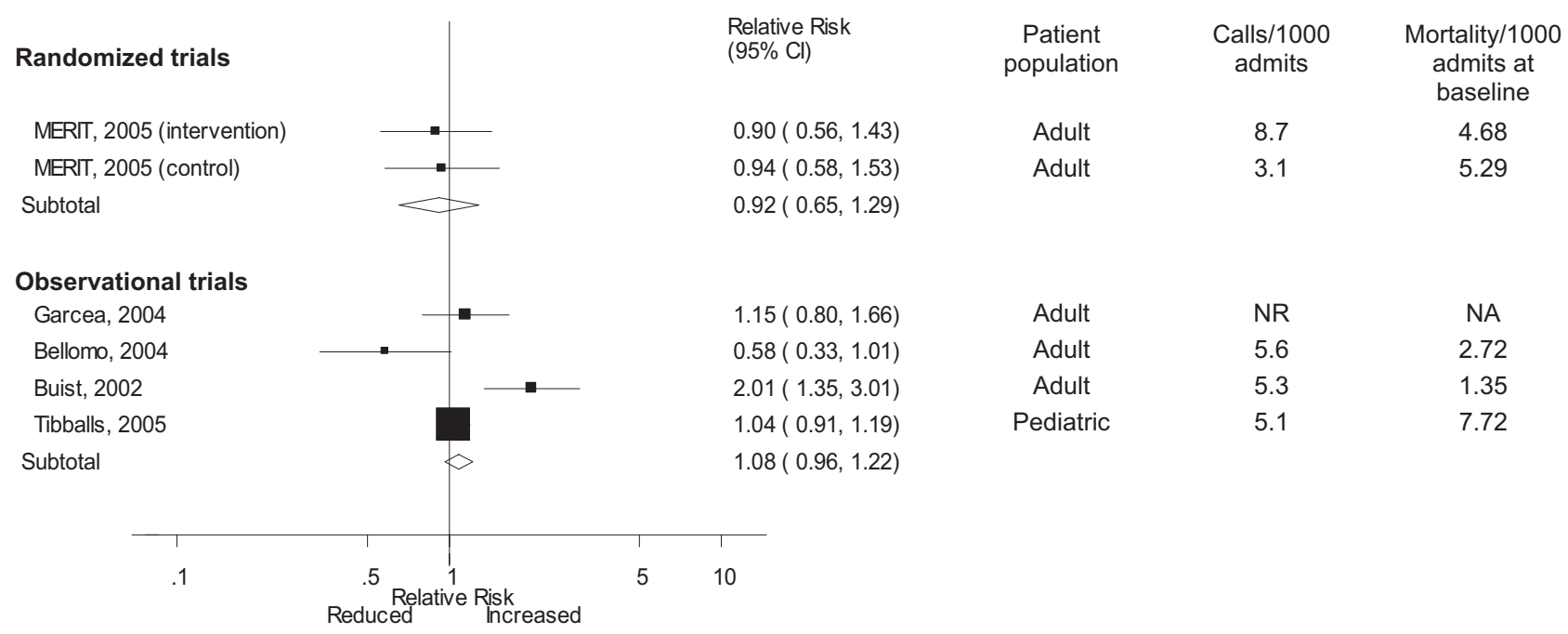

FIGURE 4. Effect of RRS on unscheduled ICU admissions The forest plot shows the relative risk of an unscheduled ICU admission after implementation of RRS. As shown in Figures 1 and 2, the MERIT trial intervention and control groups were treated as separate before-after trials. The study by Garcea et al. ${ }^{3}$ evaluated the effect of RRS on readmissions to ICU. The supplied outcomes are for unscheduled readmissions to ICU; thus, the baseline unscheduled ICU admission rate is not reported.

ing other QI interventions, that could influence the effect of an intervention. ${ }^{26}$ The statistically significant reduction in impatient mortality in the control arm of the MERIT trial is an instructive example; this decline could have been a result of the educational intervention on caring for deteriorating patients, other ongoing QI projects at the individual hospitals, or simply random variation during the relatively short (6-month) follow-up period. Such factors could also entirely account for the impressive results seen in the initial uncontrolled RRS studies. Nearly all the studies we reviewed also did not discuss any aspects of the hospital context that could influence outcomes for critically ill patients, such as the nurse-staffing ratio, ${ }^{43}$ ICU bed availability, ${ }^{44-46}$ overall hospital census, ${ }^{47}$ or availability of 
intensivists ${ }^{48}$ or hospitalists. ${ }^{49}$ Failure to control for-or at least report important aspects of-the environment in which the intervention was performed is akin to failing to report baseline patient comorbidities or concurrent therapies in a study of a drug's effectiveness.

Our review also suggests how bias in the measurement of clinical outcomes may have contributed to the apparent effect of RRSs. In 1 beforeafter study, patients for whom RRS activation resulted in a change code status to do not resuscitate (DNR) were excluded from calculations of mortality, ${ }^{22,50}$ resulting in underreporting of mortality after RRS implementation. Disposition of such patients was unclear in 3 other studies. ${ }^{3,36,40}$ Some studies ${ }^{22,34}$ defined cardiopulmonary arrest as any activation of the code blue team, regardless of whether the patient was actually in cardiac arrest. This almost inevitably would result in fewer arrests after implementation of the RRS, as the indications for calling the code blue team would be narrower. Finally, nearly all studies used trends in nonobjective primary outcomes (eg, unplanned ICU transfer) to support RRS effects but did not validate any of these outcomes (eg, how often did reviewers agree an ICU transfer was "preventable"), and none of the assessors of these outcomes were blinded.

Some have attributed the MERIT trial not finding the RRS beneficial to inadequate implementation, as the RRS calling rate of 8.7 calls per 1000 admissions was less than the 15 calls per 1000 admissions cited as optimal in a "mature" RRS. ${ }^{51}$ However, published studies generally reported a calling rate of 4-5 calls per 1000 admissions, ${ }^{22,23,37}$ with only 1 trial reporting a higher calling rate. ${ }^{34}$

A recent commentary ${ }^{20}$ and a systematic review of critical care outreach teams ${ }^{21}$ both addressed the effectiveness of RRSs. We sought to examine the effects of all RRS subtypes and using quantitative analysis and analysis of methodological quality, to determine the overall effect of RRSs. The results of our analysis (which included data from several newer studies $^{31,38,39}$ ) support and extend the conclusion of prior reviews that RRSs, although a potentially promising intervention, do not unequivocally benefit patients and are not worthy of more widespread use until more evidence becomes available. Our analysis also demonstrates that many studies widely cited as supporting wide implementation of RRSs are flawed and probably not generalizable.
Despite these caveats, RRSs remain an intuitively attractive concept and may be of benefit at some hospitals. Further studies in this area should focus on identifying which patient populations are at high risk for clinical decompensation, identifying the role of clinical structures of care (eg, nursestaffing ratio, presence of hospitalists) in preventing adverse outcomes and determining which specific RRS model is most effective. As well, more information is needed about educating bedside staff and RRS team members, as this is likely critical to success of the team. Unfortunately, only the article by King et al. ${ }^{31}$ provided sufficient detail about the implementation process to assist hospitals in planning an RRS. The remaining articles had only scant details about the intervention and its implementation, a common problem noted in the quality improvement literature. ${ }^{42,52,53}$

Our analysis had several limitations. We attempted to identify as many RRS trials as possible by searching multiple databases and reviewing abstract proceedings, but as the RRS literature is in its infancy, we may not have located other unpublished studies or "gray literature." There is no validated system for evaluating the methodological strength of nonrandomized studies; therefore, we assessed study quality on the basis of prespecified criteria for internal and external validity. Finally, we found significant statistical heterogeneity in our quantitative analyses, indicating that the variability between individual studies in treatment effects was greater than that expected by chance. As the primary reasons we conducted a meta-analysis was to compare the results of before-after trials with those of the randomized MERIT trial, we did not further explore the reasons for this heterogeneity, although variation in patient populations and RRS structure likely accounts for a significant proportion of the heterogeneity.

Although there is a theoretical basis for implementing a rapid response system, the published literature shows inconsistent benefits to patients and suffers from serious methodological flaws. Future studies of RRSs should attempt to define which patient populations are at risk, the essential characteristics of RRSs, effective implementation strategies, and-most important-whether any RRS improves clinical outcomes. Until such evidence is available, hospitals should not be mandated to establish an RRS and should consider prioritizing quality improvement resources for interventions with a stronger evidence base. 


\begin{tabular}{|c|c|c|}
\hline & Search terms & Citations \\
\hline 1 & $\begin{array}{l}\text { \{(rapid [ti] AND (response [ti] OR resuscitation [ti]) OR (patient at risk [ti])\} AND (program [ti] OR team* [ti] OR } \\
\text { service* [ti]) }\end{array}$ & 23 \\
\hline 2 & $\begin{array}{l}\text { medical emergency team* [ti] OR medical crisis team* [ti] OR \{(critical [ti] OR intensive [ti]) AND care [ti] AND } \\
\text { outreach [ti]\} }\end{array}$ & 87 \\
\hline 3 & hospital [ti] AND resuscitation [ti] AND team* [ti] & 11 \\
\hline 4 & medical emergency team* $\left.{ }^{*} \mathrm{ab}\right]$ OR rapid response team [ab] OR medical crisis team* $\left.{ }^{*} \mathrm{ab}\right]$ & 89 \\
\hline 5 & \#1 OR \#2 OR \#3 OR \#4 & 158 \\
\hline 6 & Resuscitation [mh] OR heart arrest [mh] OR hospital mortality [mh] & 72,488 \\
\hline 7 & $\begin{array}{l}\text { (patient care team [mh] OR critical care }[\mathrm{mh}] \mathrm{OR} \text { intensive care units [mh]) AND (patient readmission [mh] OR } \\
\text { organization and administration [mh]) }\end{array}$ & 20,321 \\
\hline 8 & $\# 6$ AND \#7 & 1,419 \\
\hline 9 & $\begin{array}{l}\text { \{(randomised[ti] OR randomized[ti] OR controlled[ti] OR intervention[ti] OR evaluation[ti] OR comparative[ti] OR } \\
\text { effectiveness[ti] OR evaluation[ti] OR feasibility[ti]) AND (trial[ti] OR studies[ti] OR study[ti] OR program[ti] OR } \\
\text { design[ti])\} OR clinical trial[pt] OR randomized controlled trial[pt] OR epidemiologic studies[mh] OR evaluation } \\
\text { studies[mh] OR comparative study[mh] OR feasibility studies[mh] OR intervention studies[mh] OR program } \\
\text { evaluation[mh] OR epidemiologic research design[mh] OR systematic }{ }^{5}\end{array}$ & $2,688,847$ \\
\hline 10 & $\# 8$ AND \#9 & 748 \\
\hline 11 & \#5 OR \#10 & 806 \\
\hline
\end{tabular}

Address for correspondence and reprint requests: Sumant R. Ranji, MD, Hospitalist Group, Department of Medicine, University of California San Francisco, 533 Parnassus Avenue, Box 0131, San Francisco, CA 94143-0131; Fax (415) 514-2094; E-mail: sumantr@medicine.ucsf.edu

Received 27 November 2006; revision received 23 February 2007; accepted 29 April 2007.

\section{REFERENCES}

1. Lee A, Bishop G, Hillman KM, Daffurn K. The medical emergency team. Anaesth Intensive Care. 1995;23(2):183-186.

2. Berwick DM, Calkins DR, McCannon CJ, Hackbarth AD. The 100000 Lives Campaign: setting a goal and a deadline for improving health care quality. JAMA. 2006;295(3):324-7.

3. Garcea G, Thomasset S, McClelland L, Leslie A, Berry DP. Impact of a critical care outreach team on critical care readmissions and mortality. Acta Anaesthesiol Scand 2004; 48:1096-1100.

4. Fletcher SJ, Flabouris A. The patient-at-risk team. Anaesthesia. 2000;55(2):198.

5. Devita MA, Bellomo R, Hillman K, et al. Findings of the First Consensus Conference on Medical Emergency Teams. Crit Care Med. 2006;34:2463-2478.

6. Cioffi J. Recognition of patients who require emergency assistance: a descriptive study. Heart Lung. 2000;29(4):262268.

7. Hillman KM, Bristow PJ, Chey T, et al. Antecedents to hospital deaths. Intern Med J. 2001;31:343-348.

8. Hillman KM, Bristow PJ, Chey T, et al. Duration of lifethreatening antecedents prior to intensive care admission. Intensive Care Med. 2002;28:1629-1634.

9. Hodgetts TJ, Kenward G, Vlachonikolis IG, Payne S, Castle $\mathrm{N}$. The identification of risk factors for cardiac arrest and formulation of activation criteria to alert a medical emergency team. Resuscitation. 2002;54:125-131.

10. Kause J, Smith G, Prytherch D, Parr M, Flabouris A, Hillman
K. A comparison of antecedents to cardiac arrests, deaths and emergency intensive care admissions in Australia and New Zealand, and the United Kingdom-the ACADEMIA study. Resuscitation. 2004;62:275-282.

11. Subbe CP, Kruger M, Rutherford P, Gemmel L. Validation of a modified Early Warning Score in medical admissions. QJM. 2001;94:521-526.

12. Young MP, Gooder VJ, McBride K, James B, Fisher ES. Inpatient transfers to the intensive care unit: delays are associated with increased mortality and morbidity. J Gen Intern Med. 2003;18(2):77-83.

13. Schein RM, Hazday N, Pena M, Ruben BH, Sprung CL. Clinical antecedents to in-hospital cardiopulmonary arrest. Chest. 1990;98:1388-1392.

14. Franklin C, Mathew J. Developing strategies to prevent inhospital cardiac arrest: analyzing responses of physicians and nurses in the hours before the event. Crit Care Med. 1994;22(2):244-247.

15. DeVita MA, Bellomo R, Hillman K. Introduction to the rapid response systems series. Jt Comm J Qual Patient Saf. 2006; 32:359-360.

16. Bristow PJ, Hillman KM, Chey T, et al. Rates of in-hospital arrests, deaths and intensive care admissions: the effect of a medical emergency team. Med J Aust. 2000;173:236-240.

17. Goldhill DR, Worthington L, Mulcahy A, Tarling M, Sumner A. The patient-at-risk team: identifying and managing seriously ill ward patients. Anaesthesia. 1999;54:853-860.

18. Kerridge RK. The medical emergency team: no evidence to justify not implementing change. Med J Aust. 2000;173:228229.

19. Kerridge RK, Saul WP. The medical emergency team, evidence-based medicine and ethics. Med J Aust. 2003;179:313315 .

20. Winters BD, Pham J, Pronovost PJ. Rapid response teamswalk, don't run. JAMA. 2006;296:1645-1647. 
21. Esmonde L, McDonnell $\mathrm{A}, \mathrm{Ball} \mathrm{C}$, et al. Investigating the effectiveness of critical care outreach services: a systematic review. Intensive Care Med. 2006;32:1713-1721.

22. Buist MD, Moore GE, Bernard SA, Waxman BP, Anderson JN, Nguyen TV. Effects of a medical emergency team on reduction of incidence of and mortality from unexpected cardiac arrests in hospital: preliminary study. BMJ. 2002;324:387-390.

23. Bellomo R, Goldsmith D, Uchino S, et al. A prospective before-and-after trial of a medical emergency team. Med J Aust. 2003;179:283-287.

24. Pittard AJ. Out of our reach? Assessing the impact of introducing a critical care outreach service. Anaesthesia. 2003;58: 882-885.

25. Cochrane Collaboration Effective Practice and Organisation of Care group. Available at: http://www.epoc.uottawa.ca/ inttime.pdf. Accessed August 4, 2006.

26. Shadish W, Cook T, Campbell D. Experimental and QuasiExperimental Designs for Generalized Causal Inference. Boston, MA: Houghton Mifflin; 2002.

27. Cretikos M, Parr M, Hillman K, et al. Guidelines for the uniform reporting of data for Medical Emergency Teams. Resuscitation. 2006;68(1):11-25.

28. Kerry SM, Bland JM. The intracluster correlation coefficient in cluster randomisation. BMJ. 1998;316:1455.

29. Donner A, Klar N. Issues in the meta-analysis of cluster randomized trials. Stat Med. 2002;21:2971-2980.

30. Higgins JP, Thompson SG, Deeks JJ, Altman DG. Measuring inconsistency in meta-analyses. BMJ. 2003;327:557-560.

31. King E, Horvath R, Shulkin D. Establishing a rapid response team (RRT) in an academic hospital: one year's experience. J Hosp Med. 2006;1:296-305.

32. Sebat F, Johnson D, Musthafa AA, et al. A multidisciplinary community hospital program for early and rapid resuscitation of shock in nontrauma patients. Chest. 2005;127:1729-1743.

33. Bellomo R, Goldsmith D, Uchino S, et al. Prospective controlled trial of effect of medical emergency team on postoperative morbidity and mortality rates. Crit Care Med. 2004; 32:916-921.

34. DeVita MA, Braithwaite RS, Mahidhara R, Stuart S, Foraida $\mathrm{M}$, Simmons RL. Use of medical emergency team responses to reduce hospital cardiopulmonary arrests. Qual Saf Health Care. 2004;13:251-254.

35. Priestley G, Watson W, Rashidian A, et al. Introducing critical care outreach: a ward-randomised trial of phased introduction in a general hospital. Intensive Care Med. 2004;30: 1398-1404.

36. Hillman $\mathrm{K}$, Chen J, Cretikos M, et al. Introduction of the medical emergency team (MET) system: a cluster-randomised controlled trial. Lancet. 2005;365:2091-2097.

37. Tibballs J, Kinney S, Duke T, Oakley E, Hennessy M. Reduction of paediatric in-patient cardiac arrest and death with a medical emergency team: preliminary results. Arch Dis Child. 2005;90:1148-1152.

38. Hunt EA, Shilkofski N, Rinke ML, et al. The effect of transition from a traditional code team to a rapid response team in a children's center: a before and after intervention trial [abstract]. Crit Care Med. 2005;33(12 suppl):A17.

39. Meredith A, Simpson SQ, Cleek C, Williamson T, O'BrienLadner A. Improved hospital mortality by institution of a rapid response team in a university hospital. Chest. 2005; 128(suppl S):182S.

40. Kenward G, Castle N, Hodgetts T, Shaikh L. Evaluation of a medical emergency team one year after implementation. Resuscitation. 2004;61(3):257-263.

41. Campbell MK, Elbourne DR, Altman DG. CONSORT statement: extension to cluster randomised trials. BMJ. 2004;328: 702-708.

42. Shojania KG, Grimshaw JM. Evidence-Based Quality Improvement: The State Of The Science. Health Aff. 2005;24(1): 138-150.

43. Aiken LH, Clarke SP, Sloane DM, Sochalski J, Silber JH. Hospital nurse staffing and patient mortality, nurse burnout, and job dissatisfaction. JAMA. 2002;288:1987-1993.

44. Sprung CL, Geber D, Eidelman LA, et al. Evaluation of triage decisions for intensive care admission. Crit Care Med. 1999; 27:1073-1079.

45. Strauss MJ, LoGerfo JP, Yeltatzie JA, Temkin N, Hudson LD. Rationing of intensive care unit services. An everyday occurrence. JAMA. 1986;255:1143-1146.

46. Selker HP, Griffith JL, Dorey FJ, D’Agostino RB. How do physicians adapt when the coronary care unit is full? A prospective multicenter study. JAMA. 1987;257:1181-1185.

47. Sprivulis PC, Da Silva JA, Jacobs IG, Frazer AR, Jelinek GA. The association between hospital overcrowding and mortality among patients admitted via Western Australian emergency departments. Med J Aust. 2006;184:208-212.

48. Pronovost PJ, Angus DC, Dorman T, Robinson KA, Dremsizov TT, Young TL. Physician staffing patterns and clinical outcomes in critically ill patients: a systematic review. JAMA. 2002;288:2151-2‘62.

49. Auerbach AD, Wachter RM, Katz P, Showstack J, Baron RB, Goldman L. Implementation of a voluntary hospitalist service at a community teaching hospital: improved clinical efficiency and patient outcomes. Ann Intern Med. 2002;137:859-865.

50. Subbe CP. Critical care outreach team's effect on patient outcome: other conclusions are possible. BMJ. 2004;328:347; author reply

51. The "MERIT" Trial of medical emergency teams in Australia: an analysis of findings and implications for the 100,000 Lives Campaign. Institute for Healthcare Improvement, 2006. Available at: http://www.ihi.org/NR/rdonlyres/F3401FEF2179-4403-8F67-B9255C57E207/0/LancetAnalysis81505.pdf. Accessed August 17, 2006.

52. Grimshaw J, Eccles M, Thomas R, et al. Toward evidencebased quality improvement. evidence (and its limitations) of the effectiveness of guideline dissemination and implementation strategies 1966-1998. J Gen Intern Med. 2006;21(suppl 2):S14-S20.

53. Hagedorn H, Hogan M, Smith JL, et al. lessons learned about implementing research evidence into clinical practice. Experiences from VA QUERI. J Gen Intern Med. 2006;21(suppl 2):S21-S24.

54. Moher D, Cook DJ, Eastwood S, Olkin I, Rennie D, Stroup DF. Improving the quality of reports of meta-analyses of randomised controlled trials: the QUOROM statement. Quality of reporting of meta-analyses. Lancet. 1999;354: 1896-1900.

55. Foraida MI, DeVita MA, Braithwaite RS, Stuart SA, Brooks MM, Simmons RL. Improving the utilization of medical crisis teams (Condition C) at an urban tertiary care hospital. J Crit Care. 2003;18(2):87-94.

56. Jones D, Bellomo R, Bates S, et al. Long term effect of a medical emergency team on cardiac arrests in a teaching hospital. Crit Care. 2005;9:R808-R815. 